\title{
Scattering vector mesons in D4/D8 model
}

\author{
C. A. Ballon Bayona ${ }^{a}$, Henrique Boschi-Filho ${ }^{\mathrm{b} \dagger}$, Nelson R.F. Braga ${ }^{\mathrm{b}} \ddagger$ and Marcus A. C. Torres ${ }^{\mathrm{b}} \S$

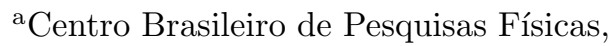 \\ Rua Dr. Xavier Sigaud 150, Urca, 22290-180 Rio de Janeiro, RJ, Brazil \\ bInstituto de Física, Universidade Federal do Rio de Janeiro, \\ Caixa Postal 68528, 21941-972 Rio de Janeiro, RJ, Brazil
}

We review in this proceedings some recent results for vector meson form factors obtained using the holographic D4-D8 brane model. The D4-D8 brane model, proposed by Sakai and Sugimoto, is a holographic dual of a semi-realistic strongly coupled large $N_{c}$ QCD since it breaks supersymmetry and incorporates chiral symmetry breaking. We analyze the vector meson wave functions and Regge trajectories as well.

\section{Introduction}

Sakai and Sugimoto proposed an elegant string model dual to large $N_{c}$ QCD at strong coupling [1. This model consists on the intersection of $N_{c}$ D4-branes and $N_{f}$ D8- $\overline{\mathrm{D} 8}$ pair of branes in type IIA string theory in the limit $N_{f} \ll N_{c}$ where $N_{c}$ and $N_{f}$ are interpreted as the color and flavor number of strongly coupled QCD. The principal characteristic of the Sakai-Sugimoto model is the holographic description of chiral symmetry breaking $U\left(N_{f}\right)_{L} \times U\left(N_{f}\right)_{R} \rightarrow U\left(N_{f}\right)$ from the merging of the D8- $\overline{\mathrm{D} 8}$ branes. This model has been used in the recent years to describe various aspects of hadron physics [12345].

In this proceedings we discuss the scattering of a photon with a vector meson using the D4D8 brane model, summarizing the results of ref 6]. One remarkable result in the D4-D8 model is the realization of an important property of hadron physics known as vector meson dominance (VMD) [7] where a hadron-photon interaction is mediated by vector mesons. As a consequence of VMD, the vector meson form factor takes the form of a sum involving vector meson masses and couplings. We first present some results for the

\footnotetext{
*email: ballon@cbpf.br

$\dagger^{\dagger}$ email: boschi@if.ufrj.br

¥email: braga@if.ufrj.br

$\S$ email: mtorres@if.ufrj.br
}

vector meson wave functions $\psi_{n}(z)$, masses and couplings and then we discuss our results for the vector meson form factor. In particular, we analyze the elastic case in which we extract the magnetic and quadrupole moments.

Form factors have also been calculated using other holographic models like the hard and soft wall model 8910 and the D3/D7 brane model [1].

\section{Vector mesons in the D4/D8 Model}

The induced metric in the probe D8-brane embedded in a D4 background can be written as 1 .

$$
\begin{aligned}
d s_{\beta D 8}^{2} & =h\left(U_{z}\right) \eta_{\mu \nu} d x^{\mu} d x^{\nu}+\frac{4}{9} \frac{U_{ß K K}}{U_{z} h\left(U_{z}\right)} d z^{2} \\
& +R^{3 / 2} U_{z}^{1 / 2} d \Omega_{4}^{2}
\end{aligned}
$$

where $U_{z}=U_{B K K}\left(1+z^{2} / U_{\beta K K}^{2}\right)^{1 / 3}, h\left(U_{z}\right)=$ $\left(U_{z} / R\right)^{3 / 2}$, the constant $R$ is related to the string length $\sqrt{\alpha^{\prime}}$ and the string coupling $g_{s}$ by $R^{3}=$ $\pi g_{s} N_{c} \alpha^{\prime 3 / 2}$ and $U_{B K K}$ is related to the KaluzaKlein mass scale by $M_{\beta K K}=3 U_{\beta K K}^{1 / 2} / 2 R^{3 / 2}$.

From the DBI action for $U\left(N_{f}\right)$ gauge fields in the D8-brane, we obtain a four dimensional effective lagrangian that can be written as [2]

$$
\begin{aligned}
\mathcal{L} & =\frac{1}{2} \operatorname{Tr}\left(\partial_{\mu} \tilde{v}_{\nu}^{n}-\partial_{\nu} \tilde{v}_{\mu}^{n}\right)^{2}+\frac{1}{2} \operatorname{Tr}\left(\partial_{\mu} \tilde{a}_{\nu}^{n}-\partial_{\nu} \tilde{a}_{\mu}^{n}\right)^{2} \\
& +M_{v^{n}}^{2} \operatorname{Tr}\left(\tilde{v}_{\mu}^{n}-\frac{g \mathcal{V} v^{n}}{M_{v^{n}}^{2}} \mathcal{V}_{\mu}\right)^{2}
\end{aligned}
$$




$$
\begin{aligned}
& +M_{a^{n}}^{2} \operatorname{Tr}\left(\tilde{a}_{\mu}^{n}-\frac{g_{\mathcal{A} a^{n}}}{M_{a^{n}}^{2}} \mathcal{A}_{\mu}\right)^{2} \\
& +\operatorname{Tr}\left(i \partial_{\mu} \Pi+f_{\pi} \mathcal{A}_{\mu}\right)^{2}+\sum_{j \geq 3} \mathcal{L}_{j},
\end{aligned}
$$

where $\tilde{v}_{\mu}^{n}$ and $\tilde{a}_{\mu}^{n}$ represent the vector and axial vector mesons, $\mathcal{V}_{\mu}$ and $\mathcal{A}_{\mu}$ are external vector and axial vector gauge fields from gauged chiral symmetry $U\left(N_{f}\right)_{L} \times U\left(N_{f}\right)_{R}$, $\Pi$ is a massless pion field and $\mathcal{L}_{j}$ represent interaction terms of order $j$. Later on we turn $\mathcal{A}_{\mu}$ off and turn on a single abelian subgroup of $U\left(N_{f}\right)$ in $\mathcal{V}_{\mu}$ that will be the source of electromagnetic interaction. The masses and couplings are defined by

$$
\begin{array}{r}
M_{v^{n}}^{2}=\lambda_{2 n-1} M_{\beta K K}^{2}, \quad M_{a^{n}}^{2}=\lambda_{2 n} M_{\beta K K}^{2} \\
g_{\mathcal{V} v^{n}}=\kappa M_{v^{n}}^{2} \int d \tilde{z} K(\tilde{z})^{-1 / 3} \psi_{2 n-1}(\tilde{z}) \\
g_{\mathcal{A} a^{n}}=\kappa M_{a^{n}}^{2} \int d \tilde{z} K(\tilde{z})^{-1 / 3} \psi_{2 n}(\tilde{z}) \psi_{0}(\tilde{z})
\end{array}
$$

where the wave functions $\psi_{n}$ are subjected to the conditions

$$
\begin{aligned}
& \kappa \int d \tilde{z}(K(\tilde{z}))^{-1 / 3} \psi_{n}(\tilde{z}) \psi_{m}(\tilde{z})=\delta_{n m}, \\
& -(K(\tilde{z}))^{1 / 3} \partial_{\tilde{z}}\left[K(\tilde{z}) \partial_{\tilde{z}} \psi_{n}(\tilde{z})\right]=\lambda_{n} \psi_{n}(\tilde{z}),
\end{aligned}
$$

where $\tilde{z}=z / U_{B K K}, K(\tilde{z})=1+\tilde{z}^{2}$ and $(6 \pi)^{3} \kappa=$ $g_{Y M}^{2} N_{c}^{2}$.

Note that the constant $g_{\mathcal{V} v^{n}}$ in (2) is the coupling of the interaction between a vector meson $\tilde{v}_{\mu}^{n}$ and an external $U(1)$ field $\mathcal{V}_{\mu}$.

Wave functions. A regularity condition for the wave functions $\psi_{2 n}(\tilde{z})$ and $\psi_{2 n-1}(\tilde{z})$ at the origin $\tilde{z}=0$, together with their parity

$$
\psi_{n}(-\tilde{z})=(-1)^{n} \psi_{n}(\tilde{z})
$$

leads to the conditions

$$
\partial_{\tilde{z}} \psi_{2 n}(0)=0 \quad, \quad \psi_{2 n-1}(0)=0 .
$$

We solve numerically the equations of motion for the vector and axial-vector modes using the shooting-method. From the normalization condition (4) and equation of motion (5) one sees that $\psi_{n}$ decrease as $\tilde{z}^{-1}$ when $\tilde{z} \rightarrow \pm \infty$. Defining $\tilde{\psi}_{n} \equiv \tilde{z} \psi_{n}$, the equation of motion takes the form

$$
\tilde{z} \partial_{\tilde{z}}\left[\tilde{z} \partial_{\tilde{z}} \tilde{\psi}_{n}\right]+A(\tilde{z}) \tilde{z} \partial_{\tilde{z}} \tilde{\psi}_{n}+B(\tilde{z}) \tilde{\psi}_{n}=0
$$
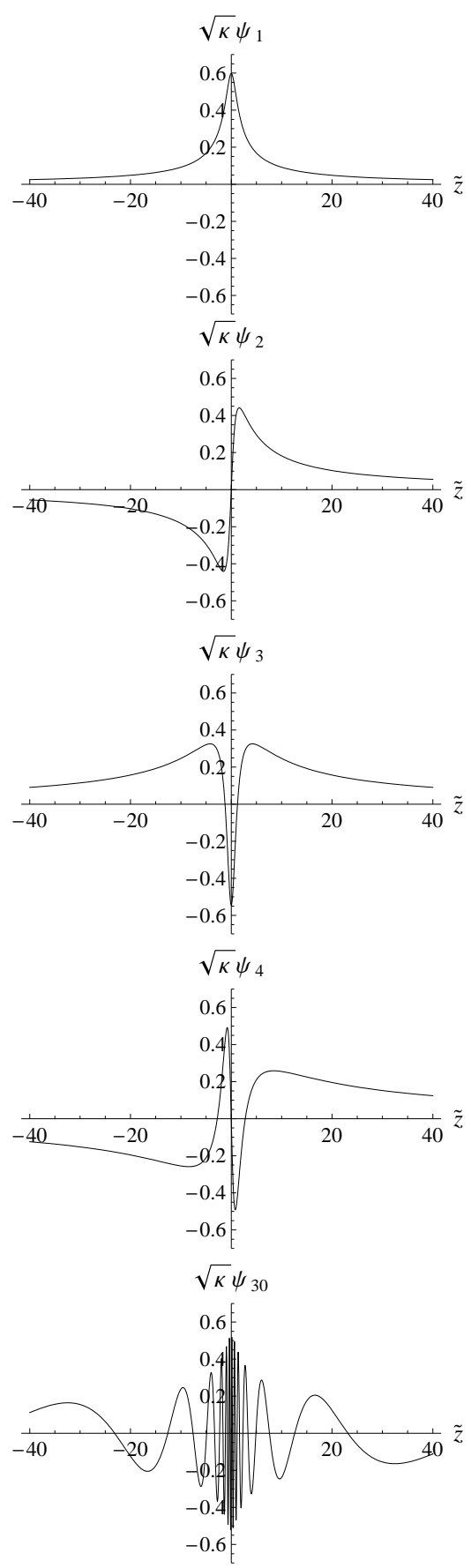

Figure 1. Wave functions $\psi_{n}(\tilde{z})$ multiplied by $\sqrt{\kappa}$ for the cases $n=1,2,3,4$ and $n=30$. 


\begin{tabular}{|c|c|c|c|c|}
\hline$n$ & $\lambda_{2 n-1}$ & $\frac{g_{v} n}{\sqrt{\kappa} M_{K K}^{2}}$ & $\sqrt{\kappa} g_{v^{n} v^{1} v^{1}}$ & $\lambda_{2 n}$ \\
\hline \hline 1 & 0.66931 & 2.10936 & 0.44658 & 1.56877 \\
2 & 2.87432 & 9.10785 & -0.14654 & 4.5461 \\
3 & 6.59118 & 20.7957 & $1.8434 ß \times 10^{\beta-2}$ & 9.00797 \\
4 & 11.79669 & 37.1502 & $-3.6885 \beta \times 10^{\beta-4}$ & 14.9573 \\
5 & 18.48972 & 58.1701 & $2.6953 ß \times 10^{\beta-4}$ & 22.394 \\
6 & 26.67017 & 83.834 & $3.0775 ß \times 10^{\beta-5}$ & 31.3182 \\
7 & 36.33796 & 114.152 & $1.8572 ß \times 10^{\beta-5}$ & 41.7297 \\
8 & 47.49318 & 148.103 & $6.9961 ß \times 10^{\beta-6}$ & 53.6285 \\
9 & 60.1312 & 188.695 & $3.5081 ß \times 10^{\beta-6}$ & 67.0149 \\
\hline
\end{tabular}

Table 1

Dimensionless squared masses and coupling constants, where $\kappa=\left(g_{Y M} N_{c}\right)^{2} / 216 \pi^{3}$.

where $A(\tilde{z})$ and $B(\tilde{z})$ are well behaved for large $\tilde{z}$ :

$$
\begin{aligned}
A(\tilde{z}) & =-\frac{1+3 \tilde{z}^{-2}}{1+\tilde{z}^{-2}} \equiv \sum_{\ell=0}^{\infty} A_{\ell} \tilde{z}^{-2 \ell / 3} \\
B(\tilde{z}) & =2 \frac{\tilde{z}^{-2}}{1+\tilde{z}^{-2}}+\lambda_{n} \tilde{z}^{-2 / 3}\left(1+\tilde{z}^{-2}\right)^{-4 / 3} \\
& \equiv \sum_{\ell=0}^{\infty} B_{\ell} \tilde{z}^{-2 \ell / 3}
\end{aligned}
$$

Using the ansatz $\tilde{\psi}_{n}(\tilde{z})=\sum_{\ell=0}^{\infty} \alpha_{\ell} \tilde{z}^{-2 \ell / 3}$ one finds

$$
\begin{aligned}
\alpha_{\ell} & =\left(\frac{4}{9} \ell^{2}+\frac{2}{3} \ell\right)^{-1} \\
& \times\left[\frac{2}{3} \sum_{k=1}^{\ell-1} k \alpha_{k} A_{\ell-k}-\sum_{k=0}^{\ell-1} \alpha_{k} B_{\ell-k}\right]
\end{aligned}
$$

with $\alpha_{0}=1$ and $\alpha_{1}=-(9 / 10) B_{1}$. Imposing the parity condition and the asymptotic behavior, we find numerically the wave functions $\psi_{n}(z)$ and their eigenvalues $\lambda_{n}$. If we go to the limit of small $z, \psi_{n}$ simplifies to $\sin \sqrt{\lambda_{n}} z$ or $\cos \sqrt{\lambda_{n}} z$, according to their parity. For large values of $\lambda_{n}$, there will be plenty of oscillations before the wave functions $\psi_{n}(z)$ reach the $z^{-1}$ behavior. We plot some wave functions in Fig. 1,

Masses and couplings. Using numerical methods, we calculated eigenvalues $\lambda_{n}$ for $n=1$

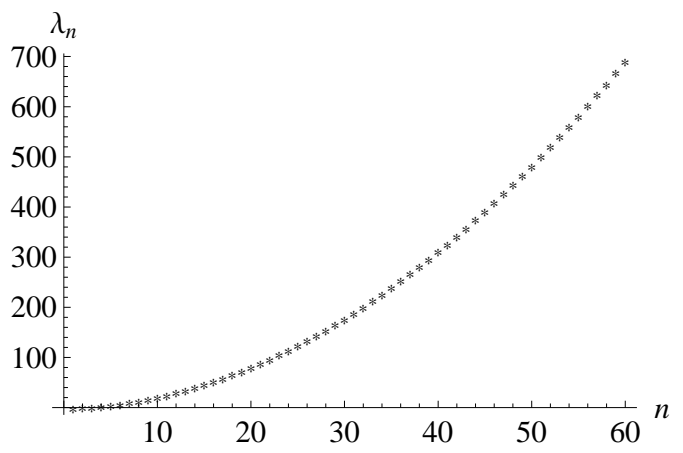

$\log \lambda_{n}$

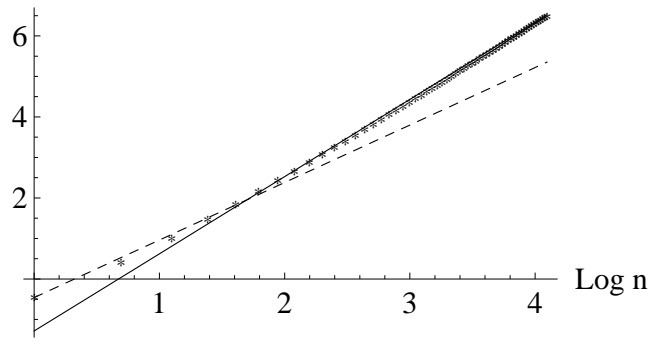

Figure 2. Meson Regge trajectory for D4/D8 model. The first graph shows the dependence of $\lambda_{n}$ with the radial number $n$. In the logarithmic graph we fit our results, the dashed line is $-0.46+1.42 \log n$ corresponding to $n=\{1,5\}$ while the solid line is $-1.28+1.91 \log n$ corresponding to $n=\{6,60\}$.

to 60 . The data for several eigenvalues are shown in Table 1 together with the calculated coupling constants for the vector mesons. With such data we obtain the corresponding Regge trajectory for the vector and axial vector mesons in the D4-D8 model. The result is shown in Figure 2. This Regge trajectory is different from that found for hadrons in the hard and soft wall holographic models [12]13]14]15.

\section{Form Factors}

The Sakai-Sugimoto D4/D8 model realizes vector meson dominance (VMD) in electromagnetic scattering. In 2] they were able to show that a 


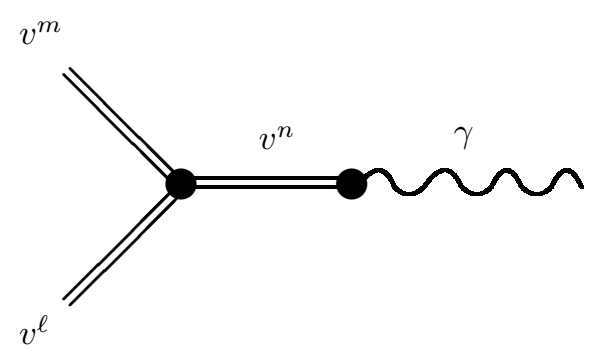

Figure 3. Feynman diagram for vector meson form factor.

photon-meson-meson couplings are all cancelled, and only a dipole interaction photon-vector meson coupling survives.

Exploring further the work done in [2], we calculate the first excitation vector meson, $\rho(770)$, elastic and non-elastic form factors derived from photon-meson scattering. We also calculated the axial vector mesons form factors [6].

\subsection{Generalized form factors}

The form factors are calculated from the matrix elements of the eletromagnetic current. The interaction of a vector meson $v^{m}$ with momentum $p$ and polarization $\epsilon$ with an off-shell photon with momentum $q=p^{\prime}-p$ is described by the matrix element

$$
\begin{aligned}
& \left\langle v^{m a}(p), \epsilon\left|\tilde{J}^{\mu c}(q)\right| v^{\ell b}\left(p^{\prime}\right), \epsilon^{\prime}\right\rangle=\delta^{4}\left(p^{\prime}-p-q\right) \\
& \times(2 \pi)^{4}\left\langle v^{m a}(p), \epsilon\left|J^{\mu c}(0)\right| v^{\ell b}\left(p^{\prime}\right), \epsilon^{\prime}\right\rangle
\end{aligned}
$$

where $\tilde{J}^{\mu}$ is the Fourier transform of the electromagnetic current $J^{\mu}(x)$. This matrix element is calculated from the corresponding Feynman diagram shown in Figure 3. We find

$$
\begin{aligned}
& \left\langle v^{m a}(p), \epsilon\left|J^{\mu c}(0)\right| v^{\ell b}\left(p^{\prime}\right), \epsilon^{\prime}\right\rangle=\epsilon^{\nu} \epsilon^{\prime \rho} f^{a b c} \\
& \times\left[\eta_{\sigma \nu}(q-p)_{\rho}+\eta_{\nu \rho}(2 p+q)_{\sigma}-\eta_{\rho \sigma}(p+2 q)_{\nu}\right]
\end{aligned}
$$

$$
\times \sum_{n=1}^{\infty} g_{v^{n}} g_{v^{m} v^{n} v^{\ell}}\left[\frac{\eta^{\mu \sigma}+\frac{q^{\mu} q^{\sigma}}{M_{v^{n}}^{2}}}{q^{2}+M_{v^{n}}^{2}}\right]
$$

where $f^{a b c}$ is the structure constant of $U\left(N_{f}\right)$ and $M_{v^{n}}$ is the mass of the vector meson $v^{n}$. Using the sum rule 2]

$$
\sum_{n=1}^{\infty} \frac{g_{v^{n}} g_{v^{n} v^{m} v^{\ell}}}{M_{v^{n}}^{2}}=\delta_{m \ell}
$$

we find

$$
\begin{aligned}
& \left\langle v^{m a}(p), \epsilon\left|J^{\mu c}(0)\right| v^{\ell b}\left(p^{\prime}\right), \epsilon^{\prime}\right\rangle=\epsilon^{\nu} \epsilon^{\prime \rho} f^{a b c} \\
& \times\left[\eta_{\sigma \nu}(q-p)_{\rho}+\eta_{\nu \rho}(2 p+q)_{\sigma}-\eta_{\rho \sigma}(p+2 q)_{\nu}\right] \\
& \times\left\{\left(\eta^{\mu \sigma}-\frac{q^{\mu} q^{\sigma}}{q^{2}}\right) F_{v^{m} v^{\ell}}\left(q^{2}\right)+\delta_{m \ell} \frac{q^{\mu} q^{\sigma}}{q^{2}}\right\}
\end{aligned}
$$

where the generalized vector meson form factor is defined by

$$
F_{v^{m} v^{\ell}}\left(q^{2}\right)=\sum_{n=1}^{\infty} \frac{g_{v^{n}} g_{v^{n} v^{m} v^{\ell}}}{q^{2}+M_{v^{n}}^{2}} .
$$

Taking into account the transversality of the vector meson polarizations: $\epsilon \cdot p=0=\epsilon^{\prime} \cdot p^{\prime}$, we find

$$
\begin{aligned}
& \left\langle v^{m a}(p), \epsilon\left|J^{\mu c}(0)\right| v^{\ell b}\left(p^{\prime}\right), \epsilon^{\prime}\right\rangle \\
= & \epsilon^{\nu} \epsilon^{\prime \rho} f^{a b c}\left[\eta_{\nu \rho}(2 p+q)_{\sigma}+2\left(\eta_{\sigma \nu} q_{\rho}-\eta_{\rho \sigma} q_{\nu}\right)\right] \\
& \times\left(\eta^{\mu \sigma}-\frac{q^{\mu} q^{\sigma}}{q^{2}}\right) F_{v^{m} v^{\ell}}\left(q^{2}\right) .
\end{aligned}
$$

Note that the term involving the factor $\delta_{m \ell}$ in eq. (14) did not contribute since in the elastic case $(m=\ell)$ we have: $2 p \cdot q+q^{2}=0$.

In a similiar way, for axial vector mesons we can calculate the form factors from the matrix element $\left\langle a^{m a}(p), \epsilon\left|J^{\mu c}(0)\right| a^{\ell b}\left(p^{\prime}\right), \epsilon^{\prime}\right\rangle$. This corresponds to evaluating Feynman diagrams similar to Fig. 3, but with the external vector meson lines replaced by the axial vector mesons $a^{m}$ and $a^{\ell}$. Note that the internal vector meson line $v^{n}$, representing vector meson dominance, is unchanged. Thus, the generalized axial vector meson form factor is

$$
F_{a^{m} a^{\ell}}\left(q^{2}\right)=\sum_{n=1}^{\infty} \frac{g_{v^{n}} g_{v^{n} a^{m} a^{\ell}}}{q^{2}+M_{v^{n}}^{2}} .
$$




\subsection{Elastic case}

The elastic form factor for vector mesons can be obtained considering the previous calculation with the same vector meson $v^{m}$ in the initial and final states. Then, from eq. (16) we find

$$
\begin{aligned}
& \left\langle v^{m a}(p), \epsilon\left|J^{\mu c}(0)\right| v^{m b}\left(p^{\prime}\right), \epsilon^{\prime}\right\rangle \\
= & f^{a b c}\left\{\left(\epsilon \cdot \epsilon^{\prime}\right)(2 p+q)^{\mu}\right. \\
& \left.+2\left[\epsilon^{\mu}\left(\epsilon^{\prime} \cdot q\right)-\epsilon^{\prime \mu}(\epsilon \cdot q)\right]\right\} F_{v^{m}}\left(q^{2}\right),
\end{aligned}
$$

where $F_{v^{m}}\left(q^{2}\right)$ is the elastic form factor:

$$
F_{v^{m}}\left(q^{2}\right)=\sum_{n=1}^{\infty} \frac{g_{v^{n}} g_{v^{n} v^{m} v^{m}}}{q^{2}+M_{v^{n}}^{2}}
$$

Note that eqs. (18) and (19) are also valid for the axial vector mesons, replacing $v^{m}$ by $a^{m}$. Using the data from Table 1] we calculate the elastic form factors for the vector meson $\rho(770)\left(v^{1}\right)$ and axial vector meson $a_{1}(1260)\left(a^{1}\right)$. We plot the results in Figure 4. Note that when $q^{2} \rightarrow 0$, the vector and axial vector form factors go to one, thanks to the sum rule (13).

For large values of $q^{2}$ we found that the form factors decrease approximately as $q^{-4}$. This can be seen in the second panel in Figure 4 where we plot the form factors multiplied by $q^{4}$. In order to explain this behavior, we expand the elastic form factors in powers of $q^{-2}$ :

$F_{v^{m}}\left(q^{2}\right)=\sum_{n=1}^{\infty} \frac{g_{v^{n}} g_{v^{n} v^{m} v^{m}}}{q^{2}}\left(1-\frac{M_{v^{n}}^{2}}{q^{2}}+\mathcal{O}\left(\frac{1}{q^{4}}\right)\right)$.

From this expansion we see the that the $q^{-4}$ term dominates for large $q$ if the following conditions hold

$$
\sum_{n=1}^{\infty} g_{v^{n}} g_{v^{n} v^{m} v^{m}}=0 .
$$

These conditions are known as superconvergence relations. For the case of $v^{1}$ we found numerically

$$
\sum_{n=1}^{9} g_{v^{n}} g_{v^{n} v^{1} v^{1}} \approx-0.0007889\left(M_{\mathrm{KK}}\right)^{2} .
$$

These results indicate that the conditions (20) are satisfied in the D4-D8 model.
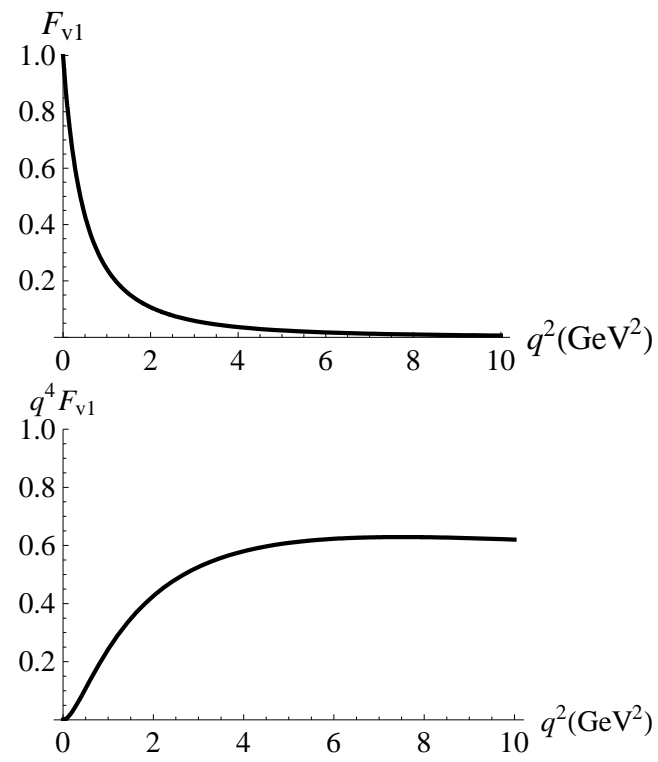

Figure 4. Elastic form factor for the $\rho$ meson.

Electric, magnetic and quadrupole form factors. The matrix element of the electromagnetic current for a spin one particle in the elastic case can be decomposed as 9

$$
\begin{gathered}
\left\langle p, \epsilon\left|J_{E M}^{\mu}(0)\right| p^{\prime}, \epsilon^{\prime}\right\rangle=\left(\epsilon \cdot \epsilon^{\prime}\right)(2 p+q)^{\mu} F_{1}\left(q^{2}\right) \\
+\left[\epsilon^{\mu}\left(\epsilon^{\prime} \cdot q\right)-\epsilon^{\prime \mu}(\epsilon \cdot q)\right]\left[F_{1}\left(q^{2}\right)+F_{2}\left(q^{2}\right)\right] \\
+\frac{1}{p^{2}}\left(q \cdot \epsilon^{\prime}\right)(q \cdot \epsilon)(2 p+q)^{\mu} F_{3}\left(q^{2}\right) .
\end{gathered}
$$

From $F_{1}, F_{2}$ and $F_{3}$ we can define the electric, magnetic, and quadrupole form factors:

$$
\begin{aligned}
F_{E} & =F_{1}+\frac{q^{2}}{6 p^{2}}\left[F_{2}-\left(1-\frac{q^{2}}{4 p^{2}}\right) F_{3}\right] \\
F_{M} & =F_{1}+F_{2} \\
F_{Q} & =-F_{2}+\left(1-\frac{q^{2}}{4 p^{2}}\right) F_{3}
\end{aligned}
$$

Then, using eqs. (18) and (22) we find that for a vector meson $v^{m}$

$$
F_{1}^{\left(v^{m}\right)}=F_{2}^{\left(v^{m}\right)}=F_{v^{m}}, \quad F_{3}^{\left(v^{m}\right)}=0,
$$


where $F_{v^{m}}$ is given by eq. (19). Hence the electric, magnetic and quadrupole form factors predicted by the D4-D8 brane model are

$$
\begin{aligned}
& F_{E}^{\left(v^{m}\right)}=\left(1+\frac{q^{2}}{6 p^{2}}\right) F_{v^{m}}, F_{M}^{\left(v^{m}\right)}=2 F_{v^{m}}, \\
& F_{Q}^{\left(v^{m}\right)}=-F_{v^{m}} .
\end{aligned}
$$

We can now estimate three important physical quantities associated with the vector mesons: the electric radius, the magnetic and quadrupole moments.

The electric radius for the vector mesons are given by

$$
\left\langle r_{v^{m}}^{2}\right\rangle=-\left.6 \frac{\mathrm{d}}{\mathrm{d} q^{2}} F_{E}^{\left(v^{m}\right)}\left(q^{2}\right)\right|_{q^{2}=0} .
$$

Using our numerical results for the form factors of the lowest excited state $\rho$, we find its electric radius:

$$
\left\langle r_{\rho}^{2}\right\rangle=0.5739 \mathrm{fm}^{2} .
$$

The magnetic and quadrupole moments are defined by

$$
\left.\mu \equiv F_{M}\left(q^{2}\right)\right|_{q^{2}=0}, \quad D \equiv-\left.\frac{1}{p^{2}} F_{Q}\left(q^{2}\right)\right|_{q^{2}=0} .
$$

Using the fact that $F_{v^{m}}$ goes to one when $q^{2} \rightarrow 0$, we obtain

$$
\mu_{v^{m}}=2, \quad D_{v^{m}}=-\frac{1}{M_{v^{m}}^{2}} .
$$

Our results for electric radius, magnetic and quadrupole moments for the vector meson $\rho$ are in agreement with the hard wall model results found in 9 .

\section{Conclusion}

We have seen that the Sakai-Sugimoto D4-D8 brane model can also be used to make predictions on the properties of the vector mesons, in particular the $\rho$ meson. These results can be extended to axial vector mesons, like the $a^{1}$ meson $[6]$.

Acknowledgements. We would like to thank the organizers of the LC2009 at ITA, SP, Brazil for their hospitality. The authors are partialy supported by CNPq, Capes and Faperj, Brazilian agencies.

\section{REFERENCES}

1. T. Sakai and S. Sugimoto, Prog. Theor. Phys. 113 (2005) 843 arXiv:hep-th/0412141.

2. T. Sakai and S. Sugimoto, Prog. Theor. Phys. 114 (2005) 1083 arXiv:hep-th/0507073.

3. H. Hata, T. Sakai, S. Sugimoto and S. Yamato, arXiv:hep-th/0701280.

4. K. Hashimoto, T. Sakai and S. Sugimoto, Prog. Theor. Phys. 120 (2008) 1093 arXiv:0806.3122 [hep-th]].

5. K. Hashimoto, T. Sakai and S. Sugimoto, arXiv:0901.4449 [hep-th]].

6. C. A. Ballon Bayona, H. Boschi-Filho, N. R. F. Braga and M. A. C. Torres, to appear.

7. J. J. Sakurai, Currents and Mesons, Chicago Univ. Press, Chicago, 1969.

8. H. R. Grigoryan and A. V. Radyushkin, Phys. Rev. D 76, 095007 (2007) arXiv:0706.1543 [hep-ph]].

9. H. R. Grigoryan and A. V. Radyushkin, Phys. Lett. B 650, 421 (2007) arXiv:hep-ph/0703069.

10. S. J. Brodsky and G. F. de Teramond, Phys. Rev. D 77, 056007 (2008) arXiv:0707.3859 [hep-ph]].

11. S. Hong, S. Yoon and M. J. Strassler, JHEP 0404, 046 (2004) arXiv:hep-th/0312071.

12. G. F. de Teramond and S. J. Brodsky, Phys. Rev. Lett. 94, 201601 (2005) arXiv:hep-th/0501022.

13. J. Erlich, E. Katz, D. T. Son and M. A. Stephanov, Phys. Rev. Lett. 95, 261602 (2005) arXiv:hep-ph/0501128.

14. H. Boschi-Filho, N. R. F. Braga and H. L. Carrion, Phys. Rev. D 73, 047901 (2006) arXiv:hep-th/0507063.

15. A. Karch, E. Katz, D. T. Son and M. A. Stephanov, Phys. Rev. D 74, 015005 (2006) arXiv:hep-ph/0602229. 